\title{
Author pre-print \\ Towards a multimodal social semiotic agenda for touch
}

Carey Jewitt

Citation: Jewitt, C. (2017) Towards a Multimodal Social Semiotic Agenda for Touch, In S. Zhao, E. Djonov, A. Bjorkvall and M. Boerils (Eds.) Advancing Multimodal and Critical Discourse Studies:

Interdisciplinary Research. Routledge: London. p. 79-93

\section{Introduction}

In this chapter I set out a multimodal social semiotic agenda for understanding touch communication practices. I outline how multimodal social semiotics can provide a framework that can be used to explore the materiality of touch, how these are shaped into touch based semiotic resources and modes, and how their take up by people to communicate is culturally and socially patterned and regulated. To do this I build on emerging multimodal studies on touch, and draw inspiration from Theo van Leeuwen's early interdisciplinary explorations of emergent modes (i.e. sound and colour) by asking how a multimodal social semiotic approach might be complemented by insights on touch from psychophysics, anthropology of the senses, and sociology. I articulate the relationships across these increasingly blurred disciplinary boundaries towards mapping the landscape of touch as 'semiotic resource' and 'mode(s)'.

\section{A general introduction to touch communication}


Touch can provide people with significant information and experience of the world. Touch is the first sense through which humans (and other animals) apprehend our environment and it is central to our development (Field, 2003). Touch is crucial for tool use (Fulkerson, 2014) and to communication: "Just as we 'do things with words' so, too, we act through touches" (Finnegan, 2014 p. 208). Indeed, knowing how to infer meaning from touch is considered to be the very basis of social being (Dunbar, 1996). Touch has many social functions in the everyday life of societies and has been honed to be a specialised form of communication within some social cultural groups. Touch is one means of enacting social relations (interpersonal meaning) and creates a stance to the world. These include greetings - shaking hands, and embracing; intimate communication - holding hands, kissing, cuddling, and stroking; and more negatively in correction - punishment, or restraining. Touch is commonly used to communicate emotions and has a role in communicating complex social messages of trust, receptivity, and affection as well as nurture, dependence, and affiliation (McLinden and McCall, 2002). Touch has been shown to be an effective means of influencing people's attitudes and creating bonds with people and places (Krishna, 2010). In clinical and professional situations, for example, interpersonal touch has been shown to improve information flow and to result in a more favourable evaluation of communication partners and to increase compliance (Field, 2010). Touch also fulfills social functions related to experiential (or ideational) meaning that serve to construct our experience of the world - providing people with information about objects, for example, interpreting texture, temperature, and perceptual understanding. 
Despite the centrality of touch to people's lives there is limited social science engagement with and understanding of touch. The neglect of touch can be tied to the social-historical value and positioning of touch within social science:

- Touch is given considerably less value and attention than sight and speech as a consequence of the (Cartesian) association of the resources of sight and speech with 'the mind' and the resources of touch with 'the body'

- Touch communication is associated with marginalised groups, including the visually and hearing impaired

- Touch does not 'belong' or 'map' to any one specific discipline (cf. language to linguistics).

One result of this relative neglect is that social science understanding of touch-based communication is less developed than that of other communicative forms (modes from a multimodal social semiotic perspective) such as speech, gesture, or gaze. Linguists have largely ignored touch, although there are few studies on it within socio-linguistics that have extended the role of hand gestures in interaction to consider touch. In his seminal book Gesturecraft (2009) Streeck explores the communicative ecologies in which hand-gestures appear. He analyses gesture as embodied communicative action grounded in the hands' practical and cognitive engagement with the material world. He argues that the hands are (with the possible exception of the eyes) the most important part of our body in "providing us with knowledge of the world, and no organ (except the brain) has played a greater part in creating the world that humans inhabit" 
(p.4). Streeck goes on to state that we need to understand gestures as physical touch, rather than visual phenomena:

Because gestures are visual phenomena for interlocutors and are often looked at and seen by the people making them, it is often falsely assumed that gesture is a medium which transforms visual experience into visual representations. Rather, as a medium of understanding, gesture incorporates haptic epistemology: it is driven by the body's practical acquaintance with a tangible environment that it has forever explored, lived in, and modified. The beholder, the recipient of conversational gesture, also draws upon an undisclosed background of haptic understandings, couplings of motorschemata and things in the world; otherwise, they would be unable to recognize the action patterns that the gestures instantiate (p.208).

Multimodality has also largely overlooked touch, with a few notably exceptions that I will return to later in this chapter. As a consequence, touch is a relatively unchartered social semiotic terrain.

\section{Turning a multimodal social semiotic lens on touch}

Multimodal social semiotics examines everyday interactions, with other social beings and / or with artefacts (this term is used to refer to semiotic materials produced by people, that is anything that bears the traces of semiotic work, e.g. an object, a digital touch device, etc.) to understand processes of meaning making. Multimodal analysis provides insight on these processes and their outcomes through micro observation and the comparison of the semiotic and modal features of an artefact or the flow of interaction in a given social moment and place. A common analytical starting point is to generate a general description of an artefact or sequence of interaction (e.g. its genre, materiality, and general structure) to locate it in the wider world of 
representation and communication, to identify and describe the modes and semiotic resources (defined below) that are available in a given situation, how people use them, the choices they make and what motivates these, and how their in situ choices are shaped by (and realise) power. Within a multimodal social semiotic framework both artefacts and interaction are positioned as semiotic material traces - the outcome of a person's or people's actions, imbued with the sign maker's interests mediated through the environment in which the sign was produced and newly encountered. In other words this approach is concerned with understanding the social world as it is represented in/through interaction and artefacts.

To theorize the contingent and fluid boundaries of 'modes of touch' I suggest that it is necessary to situate the social process of producing and using semiotic resources and modes within the bodily, material, and sensory possibilities of touch and their cultural histories. I will take sound as an illustrative example to provide a starting point for the process of exploring touch. The materiality of sound (sound waves, oscillation in pressure through air and water) in the form of vibrations travels (through air or water) to the ear or is produced via the vocal chords and diaphragm. The perception of these vibrations is linked to the physiological, psychological, and neurological capacity of a person (or an animal). The experience of sound at this level is named as pitch, duration, loudness, timbre, spatial location, and sonic texture (van Leeuwen, 1999). We can use this approach to explore the materiality of touch, its take up, and the dimensions of how it is experienced. How and what specific meaning functions these elements come to mean (that is, semiotically), is shaped by human sensory capacities in concert with the material potentials of sound or touch etc. and their use in specific social contexts. In other words the semiotic meanings of elements of sound such as pitch or loudness come to have situated social cultural meanings - rather than being universal or static meanings. What is classified and dismissed as 
'noise' (unwanted sound) or celebrated as 'sound' (what included/excluded?) is culturally situated, not universally the same. A multimodal social semiotic approach can contribute to the exploration of touch, by examining what is counted as touch by a social group in a given context and what semiotic meanings are associated with the dimensions of touch (e.g. location, duration, or pressure). For instance, to place one's hand on the shoulder of another person, to hold it there for a long time, with pressure, can communicate intimacy and reassurance, or power and control. Modes come about by the regularization and organisation of sets of semiotics resources to realize Halliday's three 'metafunctions' (ideational, interpersonal, intertextual meanings). It is important to note with regard to the relationship between sense and mode: sense does not map directly in a 1:1 way to mode. The aural sense is a basis of sound and its modal shaping into, for example, speech, music, and sound effect. Similarly, touch as sense, and the physical dimensions of touch are shaped to realise different modes: I will return to this later in the chapter in more detail. It is productive to start to map the modal qualities, materiality and semiotic potential of emergent touch based modes, and to ask under what social conditions and in what social contexts are touch-based resources shaped through their use by people to become semiotic resources or fully articulated modes? What do people use touch to achieve in multimodal complexes? Who uses them? And how are they used and what established conventions inform their use? In the case of touch, this might take us from the contexts of therapy (e.g. massage) to the specialist touch of crafts (e.g. ceramics) to tactile hand signing (e.g. Pro-Tactile ASL) for DeafBlind communities. Finally, multimodal social semiotics is concerned with how technologies re-shape semiotic resources, modes and practices through their digital production, broadcasting/dissemination, and consumption. At this point in time the touch communication technologies are at an early stage in their development, the digital remediation herald new possibilities and practices for touch. 
In the remainder of this chapter I propose a multimodal social semiotic agenda for touch communication to:

- Describe and document the materiality of touch and how these are experienced

- Map the semiotic resources and affordances of touch

- Identify and examine touch-based modes: their semiotic principles and meaning potentials

- Explore who can touch: agency and power

- Characterise people's use of touch for communication with attention to the cultural and social norms and power relations that shape their use.

In doing so I argue for the benefits of complementing multimodal analysis with social research on the physical, material, sensory possibilities and cultural histories of touch.

Social semiotics has been used to develop detailed and systematic descriptive frameworks for the analysis of images (Kress and van Leeuwen, 2006), colour (Kress and van Leeuwen, 2002; van Leeuwen, 2010), and sound (van Leeuwen, 1999) among other modes. While there is some debate as to whether modes are experienced and (perceived) interpreted as singular they are usually encountered as components of multimodal complexes, and have many shared semiotic features and principles, hence the term 'multimodal'. Nonetheless, there is value in focusing in on a mode, in this chapter - touch, to understand its particular features and functions. To date there has been little multimodal attention on touch - either in relation to specific touch-based 
mode(s) or to touch as a part of a multimodal ensemble. The research that has been conducted can be characterised as exploratory and theoretical.

\section{Documenting the materiality of touch}

Within multimodality the body is understood as a semiotic resource for meaning making. Multimodality pays analytical attention to how people use and interpret specific modes (e.g. gesture, gaze, posture, movement) and how these interact to represent and communicate meaning

(e.g. about a person's identity). Stein (2012:26) working within the multimodal semiotic tradition, argues that the body and the senses are integral to multimodal communication:

The materiality of semiotic modes is related to the sensory possibilities of the body [...] The concept of multimodality is inseparable from bodies. Bodies produce multimodality through how they are constituted sensually and how these senses act on the world and are acted on.

Materiality can be physical - marks, textures, shapes and forms - in the sense that it can be felt, heard or touched: in this way we can understand materiality as a direct 'interface between the natural and cultural world' (Stein, 2012 p. 26). However, materiality can also have a nonphysical appearance, such as spoken words or notes in which sounds have been culturally worked on to produce particular aural modes, speech and music. Stein connects the material what Kress (2010) refers to as 'stuff' - that is what a society makes available to be shaped into semiotic means for the expression of its meanings, with the sensory capacities of people to 'take up' or 'take in' these meanings. This brings the physical and sensory body clearly into view and complements a multimodal social semiotic perspective by re-focusing attention on how 
materiality and 'stuff' is brought into the social domain and socially worked into semiotic resources and principles. In doing so I seek to connect multimodality with the senses and processes of perception, domains of the biological, psychological and neurological that are generally seen as sitting outside of the concerns of multimodality and social semiotics. Understanding the physical, material, and sensory aspects of touch are a part of when and how touch-based resources are taken up (or excluded) and how they can shape - or are shaped by people to become semiotic resources. Multimodality can draw insights on the dimensions of touch from a psycho-physical and neuropsychological account of the physical experience and perception of touch and its methodological focus on the skin as an organ, its sensory receptors (nerve endings and corpuscles), and the somatosensory area of the brain (using EGC, Galvanic Skin Tests, MRIs, and neuroimaging technologies) (Spence, 2013). These accounts are limited through their focus on the individual and understanding of the senses as fixed and universal, and not recognising "the role that culture plays in the modulation of perception senses function" (Howes, 2010, p.161). While sensation is more than a biological process, insights on the physical dimensions of touch and the physiological processes through which 'signals' or tactile sensations (e.g. pain, temperature, pressure) are perceived are the 'stuff' of semiotics.

\section{Mapping the semiotic resources and affordances of touch}

As already noted, a common starting point for a multimodal social semiotic analysis is to identify and describe the semiotic resources that are available in a given situation, and how people choose and use them. 'Semiotic resources' is a term used to refer to the meaning potential of material 
resources, which developed and accumulated over time through their use in a particular community and in response to certain social requirements of that community. Affordance, when used in social semiotics, is a term that refers to the idea that different modes offer different potentials for making meaning. Modal affordances affect the kinds of semiotic work a mode can be used for, the ease with which it can be done, and the different ways in which modes can be used to achieve broadly similar semiotic work. Modal affordances are connected both to a mode's material and social histories, that is, the social purposes that it has been used for in a specific context.

The example I present here shows how semiotic resources can serve as a multimodal social semiotic entry point for touch and to expand our limited terms to describe touch and tactile experiences. Djonov and van Leeuwen (2011) conducted an exploratory study of the meaningmaking potential of 'tactile surface texture' and 'visual texture', in the presentation of texture (as a fill option for shapes and backgrounds) in PowerPoint from 1992 to 2007. I include this somewhat tangential study in this chapter as it demonstrates the usefulness of examining semiotic resources in the context of 'tactile experience'. The study explored how tactile sensation can translate into meaning through a focus on the materialization of texture in different media and "developing parameters for describing tactile and visual surface texture" (ibid: 542)and their meaning-making potentials through the exploration of three factors.

First, the provenance of texture, that is, the question of where a signifier 'comes from', comes to be associated with values and meaning. They use the example of 'denim' a material with a distinct texture which is associated with blue jeans, the heavy duty trousers for labourers and 
came to be used to signify an 'imagined identification with' and assigned the values of "American cowboys and pioneers, a preference for simplicity and functionality, a choice of equality and against class society" (ibid: 546).

Second, experiential meaning potential which is a meaning potential that is based on people's prior physical, bodily experience of, in this instance a texture. For example, the "rough and coarse" texture of denim, qualities which "depending on the context of interpretation [...] can receive positive or negative interpretations" (ibid: 547). Mapping the experiential meaning potential of texture involves:

Extracting the qualities that will allow a given texture to be described and compared with others. Such mapping is a sensory exploration which not only identifies what these qualities are, but also how they are associated with one or more different senses. (ibid 548)

They explored the experimental meaning potential of tactile texture - that is texture that can be sensed by moving a finger lightly over the surface of an object and which can also involve shape, volume and weight; and proposed that "analyzing tactile sensations into their components can help us understand how tactile sensation translates into meaning" (ibid: 548). The study of semiotic perceptions is both subjective and comparative, because a person

brings to consciousness the sensations that accompany the human act of feeling textural qualities [...] By feeling, with our fingertips, a large number of material surfaces and asking, not 'is it soft?' but 'is it softer or harder than other similar surfaces?', by recording and describing our observations $[\ldots]$ and by benchmarking them against the material qualities described in the literature (ibid: 549). 
Building on this exploration of texture a system network of six primary qualities was designed to describe tactile surface texture as 'clines' or matters of degree rather than as 'binary opposites': liquidity from wet to dry; viscosity from sticky to non-sticky; temperature from hot to cold; relief from flat to relief; density from dense to sparse; rigidity from soft to hard.

The meaning of each of these qualities is "a product of the inherent qualities they represent (e.g. the idea of softness), the other textural, visual and aural qualities with which they co-articulate, and the context in which they occur and are interpreted" (ibid: 549). For example, with reference to the quality of liquidity they suggest that all textures have a value on "a scale that runs from wet to dry" and draw on their own experiences and associations of liquidity to explore its meaning potentials (e.g. wetness is linked to positive associations with 'water and life' for beauty products and foodstuffs, and negative ones with 'rot and decay'; while dryness may connote aging but also cleanliness and comfort) (ibid: 549).

Building on sensory anthropological research (Howes, 2014), I would counter that the social cultural context is central to understanding the qualities themselves. That is that what counts as touch, both as a sense and as it is socially worked into a mode varies and is culturally specific, and historically fluid. This example shows how a focus on material and semiotic qualities, resources and affordances can help to get at touch. In the context of digital touch communication for instance a focus on semiotic resources and affordances could be used to generate a descriptive inventory of the resources and types of touch made available. 


\section{Identifying and exploring touch-based modes}

The question of how touch as a sense is socially shaped to become touch-based modes, and when touch is (or can be) considered a mode is a productive starting point for a multimodal social semiotic agenda for touch communication. Within a multimodal social semiotic approach what counts as a mode is: a set of semiotic resources with a regularity of use (i.e. 'a grammar') that fulfils the communication purposes (meaning functions) of that community (Kress, 2010). Returning to the example of sound, for instance, sound is not a mode rather it is the material realization of modes. In other words, sound has been shaped through people's social usage to produce a variety of modes: sound as speech, sound as music, etc. A key way that Kress and other social semioticians establish whether or not something is a mode is to ask whether it can realise the three 'Hallidayan' semiotic (meta) functions, namely to deal with interpersonal, ideational and textual meanings. Using this modal 'test', Bezemer and Kress (2014) expectation is that for touch to become the material basis of a mode, to be considered a mode, it needs to be able to realise meanings in the three meta-functions. It is important to note, however, that within this broad expectation, each mode differs in its materiality, semiotic features, and cultural histories and, therefore, how and what meanings are realised in different modes also differs across modes. Applying these criteria touch is clearly already a mode:

(a) Touch is designed for one or more specific others and someone is addressed (e.g. a handshake); this meets Halliday's interpersonal metafunction

(b) Touch communicates something about the world (e.g. touching an object to bring it 
into the realm of attention, to show its temperature or texture); this meets Halliday's ideational metafunction

(c) Touch is coherent with signs made in the same and other modes in forming a complete semiotic entity, an interaction (e.g. a handshake accompanied by saying 'nice to see you again'); this meets Halliday's (inter)textual function.

At least it is a mode for certain social groups - e.g. 'tactile signing' also known as hand signing or more recently Pro-Tactile ASL. However, Bezemer and Kress (2014, p.80) suggest that:

We can distinguish between communities in which touch is weakly developed, has limited semiotic reach or 'communication radius' and communities in which touch has been developed into a mode which is highly articulated, with extensive reach.

The question of whether and when touch can be considered a mode resonates with early multimodal social semiotic explorations of sound as the material realisation of mode (van Leeuwen, 1999) and colour (Kress and van Leeuwen, 2002). This question has been taken up to explore touch in a range of contexts (see Walsh and Simpson, 2014) including touch in the context of learning with iPads (Crescenzi, Jewitt and Price, 2014; Price, Jewitt and Crescenzi, 2015). In some social contexts people's usage of colour and sound fitted the definition of a fully articulate mode; in others they exhibited 'mode-like' qualities and potentials when used in combination with other modes. The same appears to be the case for touch, at least this moment in time. However, I anticipate that this will change through technological innovation that will extend and reconfigure touch capacities and practices. 


\section{Exploring who can touch: interest and agency}

Who and what can be considered to have the capacity to touch (or respond to touch) is significant to exploring touch.

Whilst accepting touch can be a mode, Bezemer and Kress (2014) question what counts as communication with respect to touch, and the interconnected question, who or what can be counted as a communicator and to whom. They distinguish between touch as a resource for 'inward' meaning making and touch as 'outward' meaning making - they classify the later as touch-as-mode. Further, they suggest two types of 'inward' touch, that is, 'implicit' or 'tacit touch' and 'explicit touch'. They use the term implicit touch to refer to types of touch that are taken for granted (e.g. kneading dough, typing, tapping links on a touch-screen). While this type of touching may be meaningful to the person touching (they can derive meaning or understanding about an object) they suggest it is not intended to represent or communicate meaning (though of course it may be interpreted as being meaningful by an observer).

Nonetheless they suggest it is not addressed to a communicational other - that is they distinguish between meaning making via touch and communication. Bezemer and Kress use the term 'explicit touch' to describe "touching to 'explore' the world - surfaces, temperature, structures, textures, and so on" (ibid, p. 78). They suggest that this has an effect on the "explorer who feels the tangible characteristics" (ibid) of the world under investigation and that as a result meaning making is involved, although they argue communication is not present because there is no addressee for the touch. For Bezemer and Kress it is only 'outward' touch that has the potential 
to meet the criteria of becoming a mode because it can be "designed as a message", "addressed" to a community, with the capacity to be "treated as having meaning" to be "interpreted" (ibid, p. 80-81): suggesting that this is touch that happens between people (or perhaps primates). I find this definition of communication as always between two people too restrictive and the demarcation between communication and meaning making to be too solid.

I argue instead for a definition of communication that considers (at least the potential of) artefacts and digital technologies as potential 'participants' in meaning making and communication. Multimodal social semiotics understands artefacts and interaction as material traces of the work of those who made them. Both artefacts and interaction are the outcome of a person's (or people's) action, imbued with their sign maker's interests as they are mediated through the environment in which the sign was produced and newly encountered. In other words the social (world) can be understood as it is represented in/through interaction and artefacts. I am not arguing that objects are agentive (as in Actor Network Theory), rather I contend that artefacts 'participate' in interaction, as they are full of meaning potentials that can be activated via interaction.

Norris (2012) offers a framing that I think may be useful for thinking this through that is underpinned by the idea of 'responsive objects'. She distinguishes between acts of 'touch', 'response', and 'feel' and notes that a sequence of touch-response-feel happens between two social actors, where a social actor may be either another human, an animal, or an object. She gives the example of two people shaking hands or a person holding the handles of a wheelbarrow walking downhill:

The (touching) social actor feels the response of the other social actor whose hand he or she is 
shaking or the pull of the wheelbarrow" (ibid, p. 8).

In a multimodal ethnographic study of horse riding she observes lessons in which a rider communicates with a horse primarily through the mode of touch. A key aspect of learning to ride is to learn how to touch the horse and how to feel the horse's response to the rider's touch. Norris explores touch via a focus on foot, leg and hand movement within the broader multimodal frame of interaction in the horse-riding lesson. This highlights that touch is a mode that can involve the whole body. She shows a sequence in which the riding instructor demonstrates both the incorrect and the correct 'touch-response-feel' expected. (This formulation shies away from equating human and horse by maintaining a focus on the human who touches, the horse who responds and the human who feels the horse's response - touch remains the purvey of the human - though the horse interprets touch.)

The potential of artefacts to participate in communication is of particular interest to research on touch as it is digitally mediated. The idea of 'responsive objects' is a feature of Cranny-Francis's work on technology and touch who suggests:

[meanings are] potentially activated when we touch [objects or others], although the nature of the particular interaction determines which meanings are deployed and to what ends.' She goes onto suggest that 'by exploring those meanings we are able to map the potentials that are available in every tactile encounter and how they might be mobilized to create the most effective and/or rich interaction. (Cranny-Francis, 2013, p. 465).

I argue that it is useful to extend touch communication to refer to contact that is human-tohuman, human-to-animal, and human-to-object (including the digital). This enables three interconnected aspects of communication to be brought into focus: 
1. The production of communicative touch artefacts: the process of producing an artefact itself is understood as a communicative one, the device is seen as designed with an imagination of its communicative context and user, and the traces of the designer's work are embedded in the design of devices as a set of meaning potentials - that are a part of shaping communication

2. Their interpretation: the ways in which people interpret these touch artefacts, what it is possible and not possible to communicate via them is an aspect of communication

3. The use of an artefact to engage with others: that is, how a user's engagement with it is constrained/shaped though not determined by its design, by their user's interests and purpose, and its the context of use.

Understanding who can touch and how touch is shaped by this condition is one part of a multimodal social semiotic agenda for touch.

\section{People's use of touch for communication: cultural and social norms}

Importantly social semiotics is concerned with issues of power and the ideological functions of modes, that is modes are understood as a part of the construction of 'reality'. This enables an analysis to explore how aspects of touch are represented as the social norm and what is placed outside of this norm, for instance how the social use of touch confers particular gendered qualities and roles. 
Classifying the social and cultural significances and meanings "generated by the embodied experience of touch" (p. 2) provided the starting point for Cranny-Francis's (2013) exploration of touch. Her focus is on how touch articulates the "values, assumptions, and beliefs of individuals and of their culture and society" (ibid, p. 2). Building on (auto-ethnographic) observations and a review of the literature she posits five 'fundamental properties of touch'.

1. Connection: That is touch creates a connection between people and objects. Touch is regulated (e.g. norms related to touch and gender, touch in a crowd) and particular meaning is given to specific touches by societies and cultures - what Cranny-Francis refers to as the 'established tactile regime of a society or culture'.

2. Engagement: is signified by 'intentional touch' between individuals as compared, for example, to unintentional touch (e.g. the contact on a crowded train that signifies connection only).

3. Contiguity: Touch signifies contiguity when we become aware of the boundary that separates us from others, objects, and the world around us.

4. Differentiation: Touch signifies the difference between the self and the other.

5. Positioning: Physically, touch creates an awareness of our location (via proprioceptive and vestibular senses) in space/time through embodied engagement with the world around us. 
These five 'properties' of touch offer a socially orientated way into thinking about touch as a social semiotic practice. I would suggest further work on the functions of touch (e.g. compliance, control and regulation, evoking memory, learning, etc.) may help to generate additional categories. Considering touch as a set of social properties is a useful starting point for the analysis of touch interactions. Cranny- Francis analyzed the visual and linguistic metaphors and narratives that people use to understand, interact with and embed touch in their everyday lives (e.g. extension, engagement, connection) to gain access to discourses about touch as well as discourses realized and communicated through touch in order to deconstruct and map their values, beliefs and assumptions about touch. The distinctions and classifications afforded by a social semiotic approach, such as those described above, serve to generate questions about the character of touch communication, ideas of intention, and processes of interpretation, as well as questions concerning the potential for touch communication with digital objects.

Research from anthropology of the senses on the sociality of the senses and ethnographic tools attuned to the sensory (Howes and Classen, 2014; Finnegan, 2014) has the potential to complement multimodal research on the cultural and historical aspects that inform the social semiotics of touch. Howes and others understand the conceptualisation and organisation of the senses as the outcome of an ideological framing related to social-cultural historical contexts. Anthropology of the senses points to the need to understand the sensory material possibilities of touch, the different sensory expressions and practices of cultures and epochs, and how communities demarcate and understand the sensorium in different ways. For example, Classen's (2012) seminal works on touch maps the cultural functions of touch to social change from the 
Middle Ages through to the current day. She maps the regulation and 'removal' of touch to changing kin relationships, the rise of the individual, the industrial revolution, the management of health and hygiene, and capitalism and points to the parallels drawn between the removal of touch and notions of civilisation. This understanding of the senses as "culturally constructed (and not always stable) categories" (Fors, Backstrom and Pink, 2013, p. 175) provides a useful backdrop to multimodal social semiotic investigation of touch.

While multimodal social semiotics provides a descriptive framework that is sensitive to power relations it can be complemented (and strengthened) by a sociological interpretation of the configurations of power that it makes visible. For instance, a social semiotic analysis of the interaction between two people may make visible (describe) that the position and posture of a man is dominant in relation to a woman and it can theorise that configuration as power, but it does not provide a theory of gender. To adequately interpret the social meaning the analyst would need to draw on additional theoretical frameworks (e.g. feminist theory, theories of power). In other words while social semiotics (multimodality) is sensitive to power it can describe how touch is being used, who is using it, what they are doing, patterns in the use of touch in a given context and therefore move towards a notion of norms of touch practices, it cannot answer the question of why they are doing it, or the social historical practices that specific touches are related to. However, despite the turn to the body and the sensory sociology has a rather patchy engagement with touch and there is a surprisingly small literature on the social aspects of touch (Linden, 2015) beyond a few classic studies linking the senses to urban living, and sociality (Simmel, 1997; Bourdieu, 1986) and exploration of 'feminine' touch (Goffman, 1979). Nonetheless social semiotic concern with power and the ideological function of modes, 
makes it a useful framework for examining how people's situated use of semiotic resources is constrained by and challenges social norms and power.

\section{Next steps}

In this chapter I have set out an emerging multimodal social semiotic agenda for touch. I have discussed the importance of not conflating touch as a sense and touch as the material realisation of mode(s), that is, touch as a social means for communicating. Nonetheless I have argued that there are important relations to be explored between them. Multimodal social semiotics provides a framework through which to explore the cultural and the social shaping of sensory resources into semiotic resources for making meaning. Using the concepts of materiality, modal affordance, and semiotic resource we can ask how are the sensory, material and physiological aspects of touch, drawn into a social system of signifiers, shaped through people's situated usage, and made to mean. That is multimodality provides a framework through which to explore the intersection (and boundaries) between the physiological, the semiotic and the social. Understanding this relationship will become increasingly pertinent in the context of touch in digital environments in which the sensory resources of touch are being newly brought into social practices and the boundaries between touch as sense, touch as mode, and technologies of touch are increasingly blurred and remade.

\section{References}

Bezemer, J., \& Kress, G. (2014). Touch: A resource for making meaning. Australian Journal of Language and Literacy, 37 (2): 77-85. 
Bourdieu, P. (1979/1986). Distinction. Cambridge: Harvard University Press.

Classen, C. (2012). The Deepest Sense: A Cultural History of Touch. Urbane: University of

Illinois Press.

Cranny-Francis, A. (2013). Technology and Touch. London: Palgrave Macmillan.

Crescenzi, L., Jewitt, C. and Price, S. (2014). The role of touch in preschool learning. AJLL, $37(2), 86-95$.

Denman, A., and Wilkinson, R. (2011). Applying conversation analysis to traumatic brain injury. Disability and Rehabilitation, 33(3), 243-252.

Djonov, E. and van Leeuwen, T. (2011). The semiotics of texture: from tactile to visual. Visual Communication 10(4), 541-564.

Dunbar, R. (1996). Grooming, Gossip and the Evolution of Language. London: Faber and Faber. Field, T. (2010).Touch for socioemotional and physical well-being: A review. Developmental Review, 30, 367-383.

Field, T. (2003). Touch. Massachusets, USA: MIT press.

Finnegan, R. (2014). Communicating. London: Routledge.

Fors, V. Bäckström, Å, \& Pink S. (2013). Multisensory Emplaced Learning: Resituating Situated Learning in a Moving World. Mind, Culture, and Activity, 20:2, 170-183, DOI:

$10.1080 / 10749039.2012 .719991$

Fulkerson, M. (2014). The First Sense: A philosophical Study of Human Touch. Massachusetts, USA: MIT press.

Goffman, E. (1979). Gender Advertisements. Boston, USA: Harvard University Press.

Howes, D., and Classen, C. (2014). Ways of Sensing: Understanding the Senses in Society. London: Routledge. 
Howes, D. (2011). Hearing scents, tasting sights: toward a cross-cultural multimodal theory of aesthetics. In F.Bacci and D.Melcher (eds.) Art and the Senses (Ch.9).Oxford: Oxford University Press.

Kress G. (2010). Multimodal Communication. London: Routledge.

Kress, G. and van Leeuwen, T. (2002). Colour as semiotic mode: notes for a grammar of colour. Visual Communication, 1 (3), 343-368.

Krishna, A. (2010). Sensory Marketing: research on the sensuality of products. London:

Routledge.

Linden, D. (2015). Touch: The Science of Hand, Heart and Mind. London: Random House. McLinden, M. and McCall, S. (2002). Learning Through Touch: Supporting Children with Visual Impairments and Additional Difficulties. David Fulton Publishers.

Norris, S. (2012). Teaching Touch/response-Feel: a first step to an analysis of touch from an interactive perspective. S.Norris (ed) (pp.2-19) Multimodality in Practice. London: Routledge. Price, S., Jewitt, C. and Crescenzi, L. (2015). The role of iPads in pre-school children's mark making development. Computers \& Education 87.C, 131-141.

Simmel, G. (1997). Simmel on Culture: Selected Writings. D. Frisby and M. Featherstone (eds.) London: Sage.

Spence, C. (2013). The multisensory perception of touch. In Bacci, F., and Melcher, D. (eds.) Art and the Senses (pp. 85-106). Oxford: Oxford University Press.

Stein, P. (2012). Multimodal Pedagogies in Diverse Classrooms. London: Routledge. Streeck, J. (2009). Gesturecraft: The Manu-Facture of Meaning. Amsterdam: John Benjamins. Van Leeuwen, T. (1999). Speech, Music, Sound. London: Routledge.

Van Leeuwen, T. (2010). The Language of Colour. London: Routledge. 
Walsh, M. and Simpson, A. (eds.) (2014). Special Issue on Touch. Australian Journal of Language and Literacy, 37(2). 